\title{
Fuel Puddle Model and AFR Compensator for Gasoline-Ethanol Blends in Flex-Fuel Engines*
}

\author{
Kyung-ho Ahn $\dagger$, Anna G. Stefanopoulou \\ The University of Michigan \\ Ann Arbor, Michigan 48109
}

\author{
Mrdjan Jankovic \\ Ford Research and Advanced Engineering \\ Dearborn, Michigan 48121
}

\begin{abstract}
Ethanol is being increasingly used as an alternative fuel to petroleum-based gasoline and diesel derivatives. Currently available flexible fuel vehicles (FFVs) can operate on a blend of gasoline and ethanol in any concentration of up to $85 \%$ ethanol (93\% in Brazil) with minimum hardware modifications. This flexibility if partly achieved through the closed-loop air-to-fuel ratio (AFR) control which maintains automatically operation around the stoichiometric ratio. Precise transient AFR control depends however on a feedforward compensator that reduces the transient effects of fuel puddle dynamics. An accurate and tunable model of the fuel puddle dynamics for gasoline-ethanol blends is, thus, necessary for the purpose of air-to-fuel ratio control. In this paper, we propose a physics-based fuel puddle model that may be used for control purposes in FFVs. In particular, the gasoline-ethanol blend is modeled using several chemical compounds and is parameterized by ethanol content. The model consists of a droplet evaporation model and a singlepuddle vaporization model. The droplet evaporation model is simulated off-line to generate port wall-impacting factors of injected fuel to be used in a single-puddle vaporization model. The single-puddle vaporization model is a cycle-based model that may be simulated on-line to characterize fuel puddle dynamics in port fuel injected engines. To verify the validity of the model, simulation results are compared with limited experimental data. A transient fuel compensator based on the proposed model is also formulated.
\end{abstract}

Index Terms-Fuel dynamics, multi-component fuel, evaporation, air-to-fuel ratio control, gasoline-ethanol blend, flex-fuel vehicles

\section{INTRODUCTION}

Currently available flexible fuel vehicles (FFVs) can operate on a blend of gasoline and ethanol in any concentration of up to $85 \%$ ethanol. The blend is denoted by the EXX nomenclature, where $\mathrm{XX}$ represents the volumetric percentage of ethanol in the blend. The United States commonly uses E85 as an alternative to the normal E0 or gasoline fuel. In Brazil, however, the fuel blend also contains water and E100 refers to a blend of $93 \%$ ethanol and $7 \%$ of water [5]. Such fuel blends mixed with the addition of water are not considered in this paper. Given the effect of fuel variation, FFVs should embed engine calibration maps in their controllers and management systems to account for this variation. One of such calibration map would account for the effect of ethanol concentration on the fuel puddle dynamics and the associated fuel injection

$\dagger$ Address all correspondence to akyungho@umich.edu.

*Funds provided from a UMICH/Ford Alliance grant. compensation for tight air-to-fuel regulation of the stoichiometric ratio of each fuel.

Air-to-fuel ratio control around the stoichiometric ratio of a fuel blend is important to meet stringent emission requirements for spark ignition (SI) engines. For a given air charge, the stoichiometric fuel is typically achieved by a combination of feedforward and feedback control on the fuel injection. The feedback controller is based on the measured ratio $(\lambda)$ of the actual air-to-fuel ratio $(A F R)$ to the stoichiometric ratio $\left(A F R_{s}\right)$ through an exhaust gas oxygen (EGO) sensor. The $\lambda$ ratio is compared to $\lambda^{d e s}=1$ and the error is used by a proportional-integral (PI) controller to adjust the fuel command. Due to the long delays in the feedback loop, most engine controllers employ a feedforward fuel command which is primarily derived from the estimated cylinder air charge divided by the assumed stoichiometric ratio of the assumed fuel blend. Furthermore, the feedforward is usually designed to eliminate the transient effects of fuel puddle dynamics in port fuel injected (PFI) engines.

Since the puddle dynamics and hence the fuel entering the cylinder depend on the ethanol content, transient fuel compensation (TFC) should be based on the gasoline-ethanol content and should retain the ability to be used in wide range of operating conditions. To this end, it is desirable to design a low-order model with a few tunable physical parameters.

A model for transient fuel compensation of gasoline in the form of a simple linear time invariant model for the fuel puddle dynamics was first introduced by Aquino in the early 1980s [1]. Curtis et al. developed the four puddle model (FPM) in which fuel films are distributed in four different locations in a PFI engine and the physics of fuel vaporization is well-exploited with use of multi-component fuel model [3]. Locatelli et al. in [10] introduced a simple control-oriented wall-wetting model based on physical principles, where the port injected fuel dynamics is modeled as uniform droplet vaporization and exponential decay of the number of airborne droplets. An extended Kalman filter is used then to identify the physical parameters of the puddle dynamics for a gasoline fuel in [11].

Batteh and Curtis introduced a fuel puddle dynamic model with alternative fuels in [2]. They used the four puddle model but unfortunately the fuel was modeled as a different combination of several fuel components for each fuel blend, i.e. indolene, E22 and E85 not allowing a continuous variation 
TABLE I

MODEL FUEL FOR GASOLINE

\begin{tabular}{|c|c|c|c|c|c|c|c|}
\hline $\begin{array}{l}\text { Component/ } \\
\text { Mass fraction }\end{array}$ & $\begin{array}{l}\text { Molecular } \\
\text { Formula }\end{array}$ & $\begin{array}{c}\text { Molecular } \\
\text { Weight }\end{array}$ & $\begin{array}{c}\text { Normal Boiling } \\
\text { Point (K) }\end{array}$ & SAE 982519 [4] & SAE 982724 [6] & SAE 2005-01-1127 [2] & This Paper \\
\hline n-butane & $\mathrm{C}_{4} \mathrm{H}_{10}$ & 58.123 & 272.7 & - & - & 0.04 & - \\
\hline iso-pentane & $\mathrm{C}_{5} \mathrm{H}_{12}$ & 72.151 & 301.0 & 0.1618 & 0.1330 & 0.16 & - \\
\hline cyclo-hexane & $\mathrm{C}_{6} \mathrm{H}_{12}$ & 84.162 & 353.8 & 0.2091 & 0.1893 & - & 0.65 \\
\hline n-hexane & $\mathrm{C}_{6} \mathrm{H}_{14}$ & 86.177 & 341.9 & - & - & 0.025 & - \\
\hline iso-octane & $\mathrm{C}_{8} \mathrm{H}_{18}$ & 114.232 & 372.4 & 0.1670 & 0.1493 & 0.32 & - \\
\hline toluene & $\mathrm{C}_{7} \mathrm{H}_{8}$ & 92.141 & 383.8 & 0.1535 & 0.1824 & 0.255 & - \\
\hline ethyl-benzene & $\mathrm{C}_{8} \mathrm{H}_{10}$ & 106.168 & 409.3 & 0.1559 & 0.1462 & - & 0.20 \\
\hline 123 trimethyl-benzene & $\mathrm{C}_{9} \mathrm{H}_{12}$ & 120.194 & 449.3 & - & - & 0.18 & - \\
\hline n-decane & $\mathrm{C}_{10} \mathrm{H}_{22}$ & 142.286 & 447.3 & 0.1171 & 0.1210 & - & 0.11 \\
\hline naphthalene & $\mathrm{C}_{10} \mathrm{H}_{8}$ & 128.174 & 491.1 & 0.0355 & 0.0319 & - & 0.04 \\
\hline n-tridecane & $\mathrm{C}_{13} \mathrm{H}_{28}$ & 184.365 & 508.6 & - & - & 0.02 & - \\
\hline ethanol & $\mathrm{C}_{2} \mathrm{H}_{6} \mathrm{O}$ & 46.069 & 351.4 & - & 0.0469 & - & - \\
\hline
\end{tabular}

within the E0-E85 range. Their model is actually not an invertible model, which was not fundamentally developed for transient compensator design.

In this paper, we present a physics-based single puddle model using multi-component model fuel for FFVs. The model is divided into two parts: droplet evaporation model to generate the wall-impacting portion of the injected fuel and the single puddle vaporization model. To capture the characteristics of the physical vaporization process, a multi-component fuel model parameterized by the ethanol content is utilized. To verify the validity of the model, model simulation results are compared with the experimental data provided in [2] for E0, E22 and E85. A transient fuel compensator using the proposed model is also formulated. Since direct pole-zero cancelation similar to the $X$ and $\tau$ pre-compensator of [12] is not possible for the nonlinear multi-component fuel puddle model, where $X$ and $\tau$ are the wall impacting factor and the fuel film vaporization time constant, respectively, introduced in [1], an approximation is used to invert the model to design a TFC. The approximation is demonstrated with a TFC simulation.

\section{Multi-Component Model Fuel}

The multi-component model fuel for gasoline can be constructed by selecting a combination of several fuel compounds which mimics the volatility of real fuel as in [6]. Table I shows such specific combinations for gasoline model listed in [4], [6] and [2] and the combination used in this paper. The mass fractions used in this paper were determined by reducing fuel components and adjusting mass fractions from the combination of mass fractions of [4] until the error between the modeled and measured [2] $\lambda$ converged to a small value after the model tuning constants were fixed to match the experimental data for the model fuel of [4]. The experimental data targeted to provide the model tuning are the $\lambda$ responses from the engine warm-up tests with several tip-ins and tip-outs as described in [2] and shown in section $\mathrm{V}$ where simulations are discussed.
A gasoline-ethanol blend is then modeled as a combination of 5 chemical compounds including ethanol. Let $e$ and $e_{m}$ denote the volume fraction and the mass fraction of ethanol in a gasoline-ethanol blend, respectively. The mass fraction $e_{m}$ is calculated by:

$$
e_{m}=\frac{e}{e+(1-e) / 1.056} .
$$

Let $f_{g, i}$ denote the mass fraction of each component for the gasoline model fuel where $i=1, \cdots, 4$ is the index representing each component, which is directly read from the last column in Table I. The mass fraction of each component in the fuel blend is then expressed as:

$$
\begin{aligned}
& f_{i}=f_{g, i} \times\left(1-e_{m}\right), \quad i=1, \cdots, 4, \\
& f_{5}=e_{m} .
\end{aligned}
$$

\section{DROPLET EVAPORATION MODEL}

It is assumed that spherical shape droplets of the same size are formed right after fuel injection. The total number of airborne droplets are modeled as an exponential decaying process [10]. The number of droplets is reduced by hitting the port wall. Droplets vaporize while they are airborne. The total mass of vaporization from droplets is regarded as the fuel mass inducted into the cylinder. A mathematical model for this process is constructed as follows. All fuel is injected as droplets of initial diameter $d_{d}^{0}$ with resulting initial droplet mass $m_{d}^{0}=\frac{\pi}{6} \rho^{0}\left(d_{d}^{0}\right)^{3}$ and initial mass of each component in a droplet $m_{d, i}^{0}=f_{i} \cdot m_{d}^{0}$, where $\rho^{0}$ denotes the initial density of mixture. Each droplet is then reduced by the droplet evaporation. Assuming stagnant air surrounding a droplet ${ }^{1}$ and fuel vapors far from the droplet surface being zero, the evolution of each droplet mass $m_{d}$ and its individual

\footnotetext{
${ }^{1}$ Although restrictive, this assumption is reasonable because fuel is injected before the intake stroke, hence, before any significant air flow surrounds the droplets.
} 


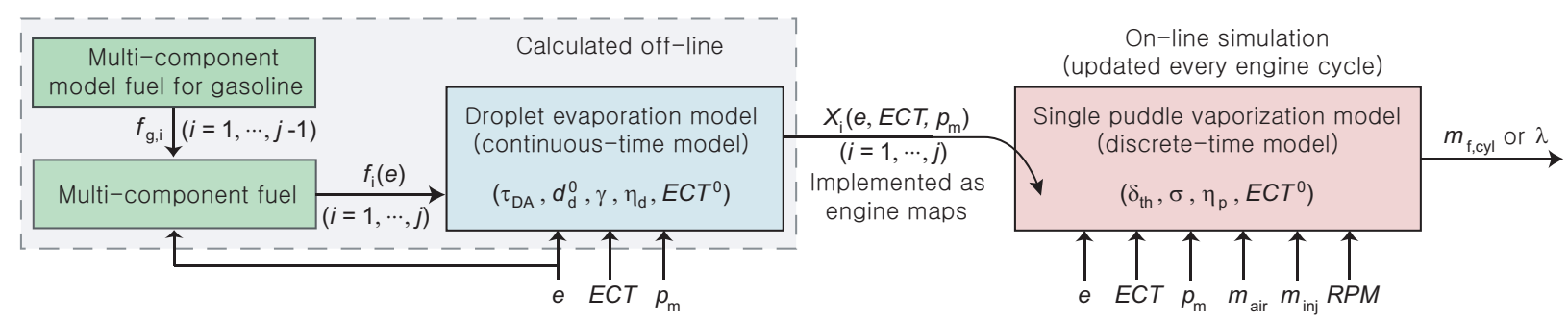

Fig. 1. Schematic of fuel puddle dynamic model for gasoline-ethanol E0-E85 blend

component masses $m_{d, i}$ are dictated by the droplet evaporation rate $\dot{m}_{E V, d}$ as in [13], [14], [18]:

$$
\begin{aligned}
\frac{d}{d t} m_{d} & =-\dot{m}_{E V, d}, \\
\frac{d}{d t} m_{d, i} & =-Y_{s, i} \cdot \frac{1+B_{d}}{B_{d}} \cdot \dot{m}_{E V, d}
\end{aligned}
$$

with

$$
\dot{m}_{E V, d}=2 \pi \cdot d_{d} \cdot \rho \cdot D \cdot \ln \left(B_{d}+1\right)
$$

where the droplet diameter follows the droplet mass reduction $d_{d}=\left(\frac{6 m_{d}}{\pi \rho}\right)^{1 / 3}, Y_{s, i}$ denotes the mass fraction of each fuel component above the droplet, $B_{d}$ is the transfer number, $D$ is diffusion coefficient of the fuel vapor, and the specific volume $1 / \rho$ is calculated as mass-weighted average of specific volumes of each fuel component. Although the experimentally observed vapor pressure for gasoline-ethanol blends deviates from the one expected from ideal mixtures as shown in [9], we neglect this complexity and use Raoult's law to our controloriented model. According to Raoult's law [16], the mass fraction of each fuel component above the droplet is expressed as:

$$
Y_{s, i}=\frac{V P_{i} \times f_{d, i}}{\sum_{i}\left(V P_{i} \times f_{d, i}\right)+P P_{a i r} \cdot M_{a i r} \cdot \sum_{i} f_{d, i} / M_{i}} .
$$

The transfer number $B_{d}$ is calculated as follows [2], [15], [16]:

$$
B_{d}=\frac{\sum_{i} V P_{i} \times f_{d, i}}{P P_{a i r} \times M_{a i r} \times \sum_{i} f_{d, i} / M_{i}},
$$

with the partial pressure of air calculated from

$$
P P_{\text {air }}=p_{m}-\frac{\sum_{i} V P_{i} \times f_{d, i} / M_{i}}{\sum_{i} f_{d, i} / M_{i}},
$$

where summation $\sum_{i}$ is performed over all fuel components, $V P_{i}$ denotes the normal vapor pressure of the $i$-th fuel component, $M_{i}$ is the molecular weight of the $i$-th fuel component, $M_{a i r}$ is the molecular weight of air $(28.97 \mathrm{~g} / \mathrm{mol}), p_{m}$ denotes the manifold absolute pressure (MAP) and $f_{d, i}$ is defined as the mass fraction of the $i$-th fuel component in the droplet:

$$
f_{d, i} \triangleq \frac{m_{d, i}}{\sum_{i} m_{d, i}}=\frac{m_{d, i}}{m_{d}}
$$

The diffusion coefficient, $D$, and the normal vapor pressures, $V P_{i}$, should be evaluated at the droplet surface temperature. However, it is hard to exactly predict the temperature. Instead, we use an apparent temperature which is parameterized by the ethanol content, $e$, and the engine coolant temperature, $E C T$ :

$$
T_{a p p, d}=E C T-(e+\gamma) \cdot \eta_{d} \cdot\left(E C T-E C T^{0}\right),
$$

where $\gamma, \eta_{d}$ and $E C T^{0}$ are three tuning constants. There are publications of fuel puddle dynamic models which include temperature dynamics of explicit states, i.e. differential equations for some temperatures, e.g. valve, cylinder and port temperatures [2], [10]. The purpose of a dynamic temperature model is to explicitly evaluate a physical property at the temperature of a designated location. However, it is still difficult to establish a simple dynamic model to predict the droplet surface temperature and a dynamic temperature model usually needs several tuning constants in the equations. A dynamic temperature model may be introduced to our model in future study. Nevertheless, in this paper, we regard a temperature change approximately as a static process parameterized by the engine coolant temperature, $E C T$, which is measured on-line. The apparent temperature does not play a role of approximating a specific temperature at a designated location. It serves as a reference temperature which gives relative distance of thermophysical properties relevant to the physical process. Using (5) and (6), (3) is expressed as:

$$
\frac{d}{d t} m_{d, i}=-\frac{V P_{i}\left(T_{a p p, d}\right) \times f_{d, i}}{\sum_{i} V P_{i}\left(T_{a p p, d}\right) \times f_{d, i}} \cdot \dot{m}_{E V, d} .
$$

Calculation of diffusion coefficient, $D$, for multi-component fuel is approximated here by mass-weighted average of diffusion coefficients of each fuel component and each coefficient may be looked up from [19] which gives functional expression in temperature. The following relation that kinetic theory would pose under ideal gas behavior assumption is also utilized [7], [8]:

$$
D \sim \frac{T_{a p p, d}^{3 / 2}}{p_{m}} .
$$

Calculation of normal vapor pressures, $V P_{i}$, can be also done by the functional expression in temperature provided in [19].

Let $X_{i}$ denote the ratio of the wall-impacting mass of $i$ th component to the total injected mass of fuel, which is the output of the droplet evaporation model and is the input to the single puddle vaporization model which will be discussed in the following section. The mass balance is then expressed as:

$$
f_{i}-X_{i}=\frac{1}{m_{d}^{0}} \int_{0}^{t_{e v}} \dot{m}_{E V, d, i} \cdot e^{-\frac{t}{\tau_{D A}}} d t .
$$


where $\tau_{D A}$ is the time constant of the decay of the number of airborne droplets, $t_{e v}$ is the time for complete vaporization of single droplet, i.e. $\left.m_{d}\right|_{t=t_{e v}}=0$. The time constant $\tau_{D A}$ is a universal tuning constant over the whole range of ethanol content, which should be significantly smaller than one engine cycle duration.

According to this model from (2)-(8), $X_{i}$ is varying in accordance with the variation of engine coolant temperature, $E C T$, manifold absolute pressure, $p_{m}$, and also with the variation of the ethanol content, $e$, i.e., $X_{i}=X_{i}\left(e, E C T, p_{m}\right)$. If we consider the effect of flow of air, then Reynolds number and Schmidt number should be involved in the formulation; in this case, $X$ may be also dependent on the air charge mass, engine RPM, and the port diameter as in [13] or [10]. Nevertheless, the stagnant air assumption may well capture the whole process of droplet evaporation or the spray injection process considering the usual timing of the fuel injection to the intake port before the intake valves open.

Note that the total wall-impacting mass fraction of the injected fuel is the sum of each $X_{i}$ over all fuel components, $X=\sum_{i} X_{i}$. The total wall-impacting mass fraction, $X$, may be used for initial rough calibration. The evolution of the droplet evaporation and the resulting wall impacting mass fractions can be computed off-line and produce a look-up table as discussed later in section $\mathrm{V}$.

\section{Single Puddle VAPORIZATION Model}

Some droplets of the injected fuel hit the port wall before their complete evaporation. That mass forms a fuel puddle on the port wall and some portion of the puddle also vaporizes from the puddle. The mass of each component in the fuel puddle at intake valve opening (IVO) is equal to the sum of the previous-cycle mass and the portion of the newly injected fuel that hits the puddle. The total puddle mass at IVO is equal to the sum of masses of each component:

$$
\begin{aligned}
m_{p, i}^{I V O}(k)= & m_{p, i}(k-1)+X_{i}\left(e, E C T, p_{m}\right) \times m_{i n j}(k), \\
& i=1, \cdots, j, \\
m_{p}^{I V O}(k)= & \sum_{i=1}^{j} m_{p, i}^{I V O}(k),
\end{aligned}
$$

where $k$ denotes the event or cycle number, $i$ is index for fuel component and $j$ is the total number of fuel components $(j=5)$. It is assumed that the rest of injected fuel is evaporated and enter the cylinder on the intake stroke. The puddle mass at intake valve closing (IVC), $m_{p}$, is derived from the Locatelli's model [10]. Assuming a cylindrical fuel puddle along the port wall, let $\delta_{t h}$ be the thickness of the puddle which is constant. The height of the puddle is varying according to the mass variation of the puddle. The vaporization rate is expressed as:

$$
\begin{aligned}
\dot{m}_{E V, p} & =\frac{\rho \cdot A_{p}}{d_{i n}} \cdot D_{p} \cdot S h_{p} \cdot \ln \left(1+B_{p}\right), \\
& =\frac{\breve{m}_{p}}{d_{i n} \cdot \delta_{t h}} \cdot D_{p} \cdot S h_{p} \cdot \ln \left(1+B_{p}\right),
\end{aligned}
$$

where $\rho$ is the density of the fuel puddle, $A_{p}$ is the surface area of the puddle exposed to air flow, $d_{i n}$ is the port diameter,
$D_{p}$ is the diffusion coefficient, $S h_{p}$ is the Sherwood number, $B_{p}$ is the transfer number and $\breve{m}_{p}$ is the instantaneous puddle mass such that $\dot{m}_{E V, p}=-\dot{\breve{m}}_{p}$. The fuel puddle mass at IVC, $m_{p}(k)$, is then obtained by integrating (16), for one cycle duration:

$$
m_{p}(k)=m_{p}^{I V O}(k)\left(1+B_{p}\right)^{-\alpha},
$$

where

$$
\alpha \triangleq C(e) \cdot \frac{120}{N} \cdot \frac{D_{p} \cdot S h_{p}}{d_{i n} \cdot \delta_{t h}} .
$$

In the above equation, $N$ denotes the engine RPM and a correction factor, $C$, parameterized by the ethanol content, $e$, is introduced to correct the effect of variation of fuel film thickness according to the ethanol content variation. The correction factor is modeled as a linear variation with the change of the ethanol content:

$$
C(e)=1-\sigma e
$$

where $\sigma$ is a tuning constant. The evaporated mass is expressed as:

$$
\begin{aligned}
m_{\text {evap }}(k) & \triangleq m_{p}^{I V O}(k)-m_{p}(k) \\
& =m_{p}^{I V O}(k)\left[1-\left(1+B_{p}\right)^{-\alpha}\right] \\
& =\bar{\alpha} \cdot m_{p}^{I V O}(k),
\end{aligned}
$$

where the mass fraction of evaporation from the mass at IVO is defined as $\bar{\alpha} \triangleq 1-\left(1+B_{p}\right)^{-\alpha}$. Sherwood number is computed as follows [17]:

$$
\begin{aligned}
u_{A \infty} & =\frac{m_{a i r} \cdot N}{120} \cdot \frac{4}{\rho_{A} \cdot \pi \cdot d_{i n}^{2}}, \\
R e_{p} & =\frac{u_{A \infty} \cdot d_{i n}}{\nu_{A}}, \\
S c_{p} & =\frac{\nu_{A}}{D_{p}}, \\
S h_{p} & =0.023 \cdot R e_{p}^{0.83} S c_{p}^{0.44},
\end{aligned}
$$

where $\rho_{A}$ denotes density of air, $\nu_{A}$ denotes kinematic viscosity of air, and $m_{a i r}$ denotes air charge mass per cycle. Let us define the mass fraction of each component in the puddle at IVO:

$$
f_{p, i}(k) \triangleq \frac{m_{p, i}^{I V O}(k)}{m_{p}^{I V O}(k)} .
$$

Let $V P_{i}$ denote the normal vapor pressure of each fuel component as used in the droplet evaporation model. The diffusion coefficient, $D_{p}$, and the normal vapor pressures, $V P_{i}$ are evaluated at another apparent temperature for the fuel puddle vaporization:

$$
T_{a p p, p}=E C T-e \cdot \eta_{p} \cdot\left(E C T-E C T^{0}\right)
$$

where $\eta_{p}$ is another tuning constant and $E C T^{0}$ is the same tuning constant used in the droplet evaporation model. Calculation of $D_{p}$ is similarly performed to the droplet evaporation 
model (11) as $D_{p} \sim T_{a p p, p}^{3 / 2} / p_{m}$. The variable $B_{p}$ is computed similar to the droplet evaporation model as follows:

$$
\begin{aligned}
P P_{a i r}(k) & =p_{m}(k)-\frac{\sum_{i} V P_{i} \times f_{p, i}(k) / M_{i}}{\sum_{i} f_{p, i}(k) / M_{i}}, \\
B_{p}(k) & =\frac{\sum_{i} V P_{i} \times f_{p, i}(k)}{P P_{a i r}(k) \times M_{a i r} \times \sum_{i} f_{p, i}(k) / M_{i}} .
\end{aligned}
$$

The computation at the step $k$ is completed by updating the masses of each fuel component at the end of the intake stroke accounting for the evaporated fuel:

$$
\begin{aligned}
m_{\text {evap }, i}(k)= & \min \left\{m_{p, i}^{I V O}(k), m_{\text {evap }}(k) \times\right. \\
& \left.\frac{V P_{i}\left(T_{a p p, p}\right) \times m_{p, i}^{I V O}(k)}{\sum_{i} V P_{i}\left(T_{a p p, p}\right) \times m_{p, i}^{I V O}(k)}\right\}, \\
m_{p, i}(k)= & m_{p, i}^{I V O}(k)-m_{\text {evap }, i}(k) .
\end{aligned}
$$

Finally, the mass of fuel inducted into the cylinder is:

$$
\begin{aligned}
m_{f, c y l}(k)= & \left(1-X\left(e, E C T, p_{m}\right)\right) \times m_{i n j}(k) \\
& +\sum_{i=1}^{j} m_{\text {evap }, i}(k) .
\end{aligned}
$$

We can compute air-to-fuel ratio, $A F R$ and $\lambda$ at each step as follows:

$$
\begin{aligned}
A F R(k) & =\frac{m_{\text {air }}(k)}{m_{f, c y l}(k)}, \\
\lambda(k) & =\frac{A F R(k)}{A F R_{s}(k)},
\end{aligned}
$$

where the stoichiometric air-to-fuel ratio is computed by:

$$
A F R_{s}=9 \times e_{m}+14.6 \times\left(1-e_{m}\right) .
$$

Fig. 1 depicts the model summary where inputs to each submodel and tuning constants associated with each submodel are shown. In the droplet evaporation model, the parameter $d_{d}^{0}$ indicates the initial droplet diameter right after injection. The droplet evaporation model passes $X_{i}$ over to the singlepuddle vaporization model as inputs. Since $X_{i}$ is an integration result according to (12), the internal states during the droplet evaporation simulation for implementation at every numerical computation time step do not have to be known. Therefore, to avoid unnecessary increase of on-line computation time, which might be caused by computing $X_{i}$ at every engine cycle, the droplet evaporation model is simulated off-line to generate $X_{i}$ and the results are implemented as engine maps in the single-puddle vaporization model which updates its states every engine cycle.

\section{Simulation}

The resulting model takes 6 inputs, $e, E C T, p_{m}, m_{\text {air }}$, $m_{i n j}, R P M$, and uses 8 parameters, $\tau_{D A}, d_{d}^{0}, \gamma, \eta_{d}, E C T^{0}$, $\delta_{t h}, \sigma, \eta_{p}$ to produce the fuel amount inducted into the cylinder and $\lambda$ as shown in Fig. 1. For parameter tuning and the model validation, the three different experiments of [2] have been obtained.
The experimental data show the $\lambda$ traces measured by a Universal Exhaust Gas Oxygen (UEGO) sensor for different fuel blends of E0, E22 and E85, respectively, while the 4.6L $2 \mathrm{~V}$ engine operates from cold start till full warm-up for each blend and several steps of tip-ins and tip-outs are applied.

As stated in section II, the multi-component model fuel for gasoline was first taken from the mass fractions in [4]. The 8 parameters associated with our model were then tuned for that model fuel which uses 8 chemical compounds including ethanol. The tuning of the model parameters is explained below. First, the initial droplet diameter, $d_{d}^{0}$, was chosen and the time constant, $\tau_{D A}$, was tuned so that the whole droplet evaporation process around the engine coolant temperature range (290-370 K) and the manifold absolute pressure range (0.1-0.8 bar) yielded a reasonable range of the wall impacting factors, $X_{i}$. The value for $E C T^{0}$ was determined to be close to

\begin{tabular}{|c|c|c|c|}
\hline Parameter & Value & Unit & Model \\
\hline$\tau_{D A}$ & $8.0 \times 10^{-5}$ & [s] & \multirow{4}{*}{ Droplet evaporation } \\
\hline$d_{d}^{0}$ & $6.0 \times 10^{-5}$ & {$[\mathrm{~m}]$} & \\
\hline$\gamma$ & 0.80 & - & \\
\hline$\eta_{d}$ & 0.31 & - & \\
\hline$E C T^{0}$ & 290 & {$[\mathrm{~K}]$} & $\begin{array}{l}\text { Droplet evaporation and } \\
\text { single-puddle vaporization }\end{array}$ \\
\hline$\sigma$ & 0.60 & - & \multirow{3}{*}{ Single-puddle vaporization } \\
\hline$\eta_{p}$ & 0.75 & - & \\
\hline$\delta_{t h}$ & $3.0 \times 10^{-3}$ & {$[\mathrm{~m}]$} & \\
\hline
\end{tabular}
ambient temperature. The product of $\gamma$ and $\eta_{d}, \gamma \cdot \eta_{d}$ was tuned to yield proper shapes of $\lambda$ excursions for E0, and then each parameter was determined by matching the $\lambda$ excursions for E85. Likewise, the film thickness, $\delta_{t h}$, was tuned to match the film vaporization rate for E0; $\sigma$ and $\eta_{p}$ were tuned to match the data for E85. In this way, true validation is obtained through the comparison between simulation and experiments for E22.

TABLE II

TUNED PARAMETERS OF SIMULATION

Table II summarizes the tuned parameters and their values. The port diameter, $d_{i n}$ is $0.045 \mathrm{~m}$. Using these parameters, the gasoline fuel components were then reduced to yield similar model simulation results to those of the previously used gasoline fuel components of [4]. Table I summarizes the optimized fuel fractions in the last column and the following simulations used those fuel fractions associated with a 4component gasoline instead of the original 7 components in [4].

Look-up tables for $X_{i}$ were built from the droplet evaporation model for $9 \times 8=72$ points with discrete variation of engine coolant temperature and manifold absolute pressure such that $E C T=290,300, \cdots, 370 \mathrm{~K}$ and $p_{m}=0.1,0.2, \cdots, 0.8$ bar prior to the puddle simulation for E0, E22 and E85. Fig. 2 and Fig. 3 depict the wall-impacting factors, $X_{i}$, for all fuel components in the multi-component model fuel and the total wall-impacting fraction, $X$, at $p_{m}=0.1,0.2, \cdots, 0.8$ bar for 

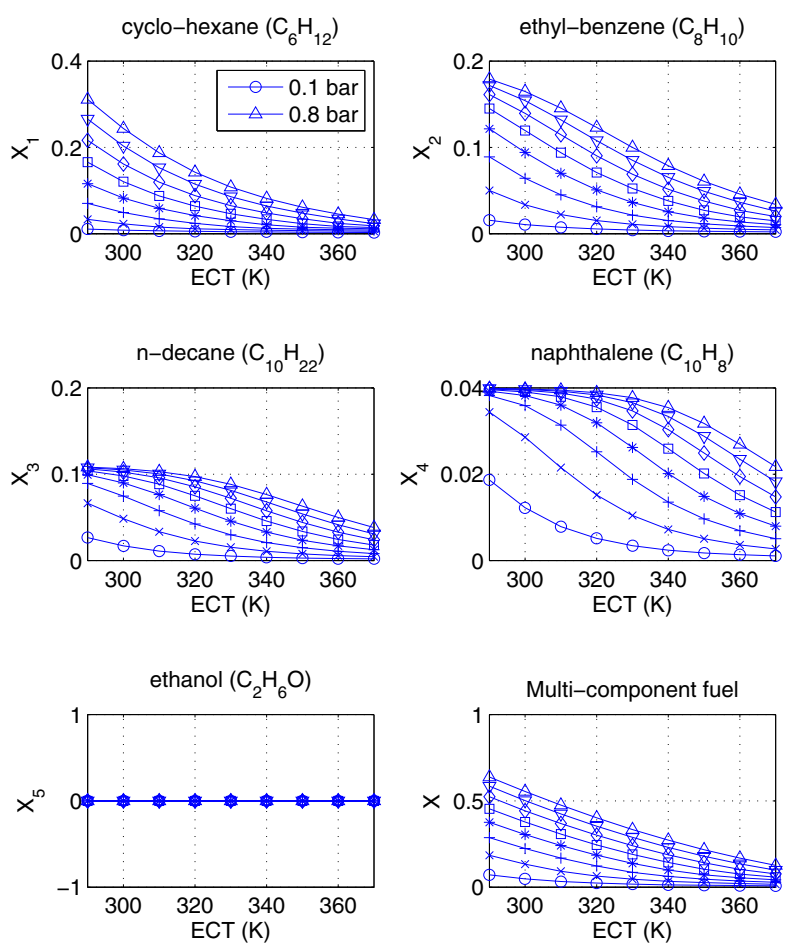

Fig. 2. Wall-impacting factor of each fuel component $\left(X_{i}, i=1, \cdots, 5\right)$ and total wall-impacting mass fraction $(X)$ for $\mathrm{E} 0$ at $p_{m}=0.1,0.2, \cdots, 0.8$ bar
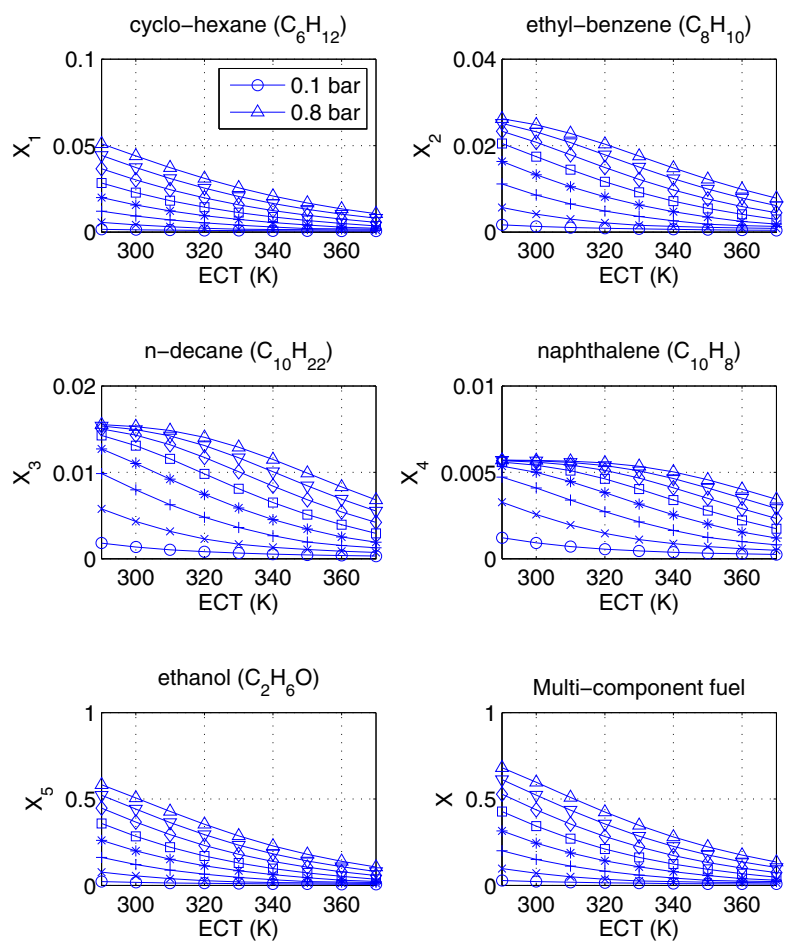

Fig. 3. Wall-impacting factor of each fuel component $\left(X_{i}, i=1, \cdots, 5\right)$ and total wall-impacting mass fraction $(X)$ for E85 at $p_{m}=0.1,0.2, \cdots, 0.8$ bar
E0and E85, respectively.

Fig. 4 shows the inputs to the (puddle vaporization) model for E0, based on the warm-up test performed in [2]. Similar inputs obtained from the tests performed in [2] were used for E22 and E85, although not shown here.
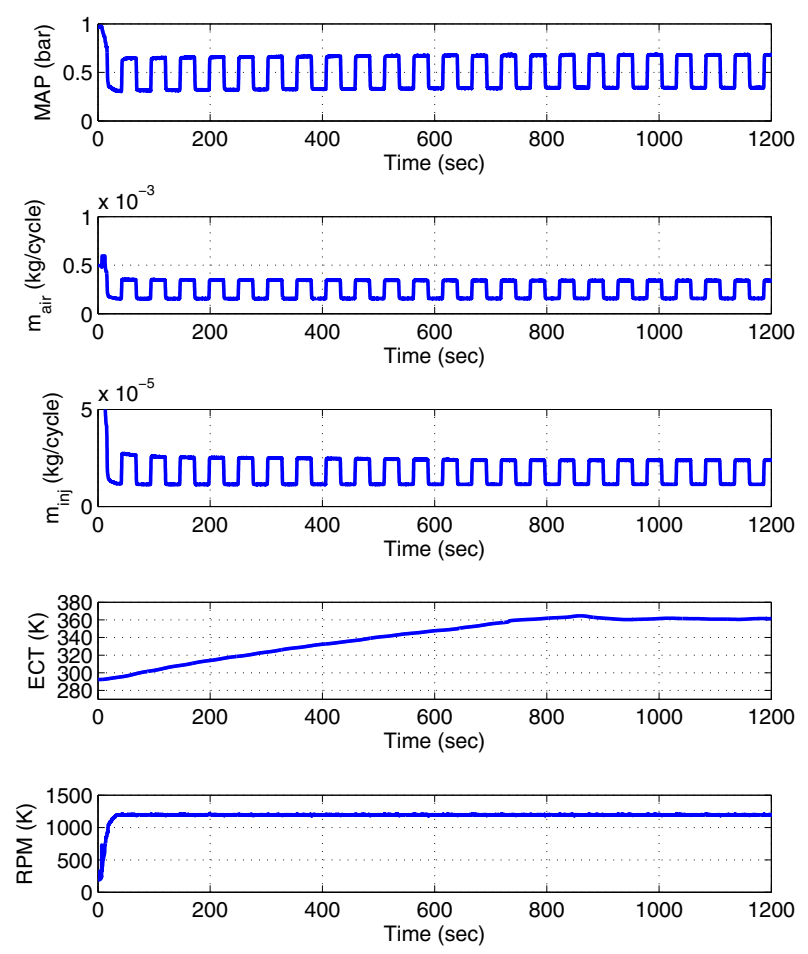

Fig. 4. Fuel injected and other measurements from [2] used for the model inputs (E0)

Fig. 5-Fig. 7 show the simulated $\lambda$ responses using the proposed model for E0, E22 and E85, respectively, compared with the experimental data measured by a UEGO sensor. The agreement between the simulations and the experimental data is quite reasonable. Model matching is especially very good for sufficiently warmed-up operations. The model also predicts the overall decrease in magnitude of the $\lambda$ excursions well. It also captures the asymmetric excursions due to tip-ins and tipouts observed in the experimental data. The verification needs to be confirmed at more operating points to evaluate the model accuracy. However, the provided simulation and experimental results reasonably support the validity of the proposed model for at least some operating conditions.

\section{TRANSIENT FUEL COMPENSATOR}

Transient fuel compensation is realized by inverting the dynamics of the fuel film and injection. This means, for a linear fuel puddle model, cancelation of pole and zero in fuel puddle dynamics with zero and pole in TFC transfer function [1], [12]. However, direct inversion of pole-zero cancelation as in a linear model is not available for our fuel puddle model. To design a transient fuel compensator, the current fuel injection, 

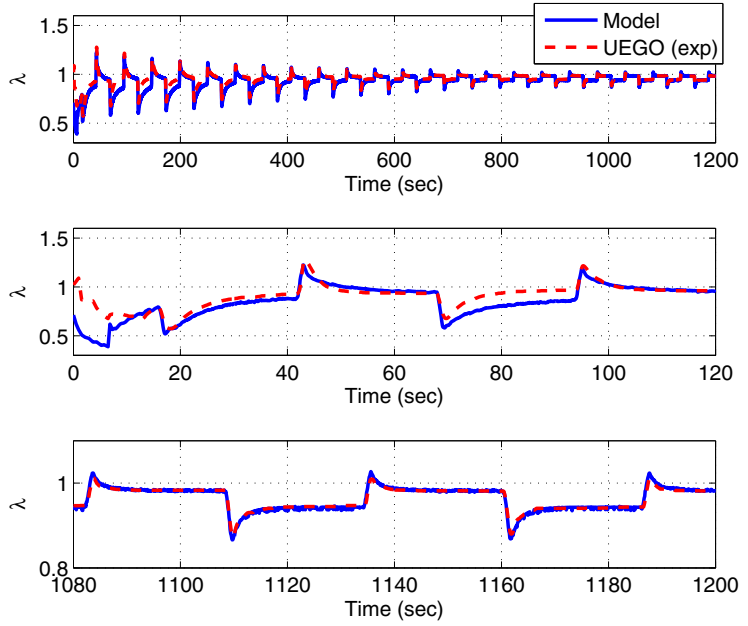

Fig. 5. Simulated and experimental $\lambda$ response for E0
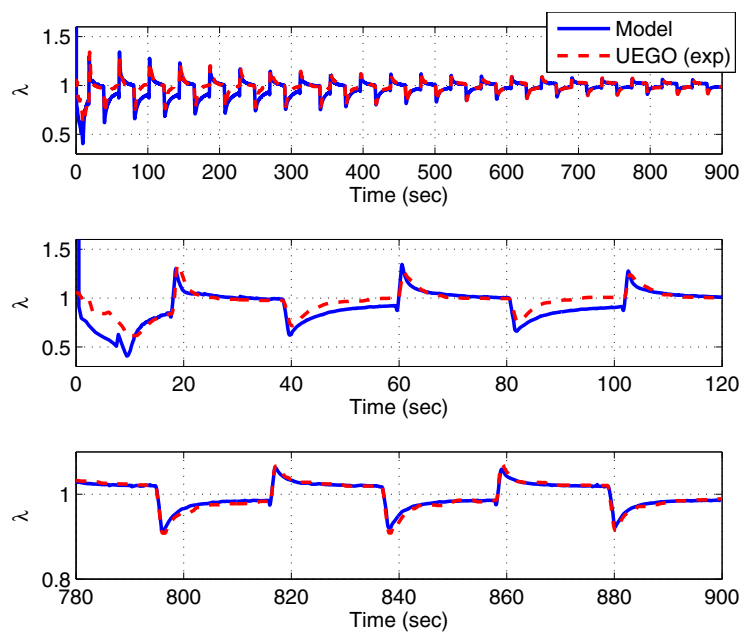

Fig. 6. Simulated and experimental $\lambda$ response for E22
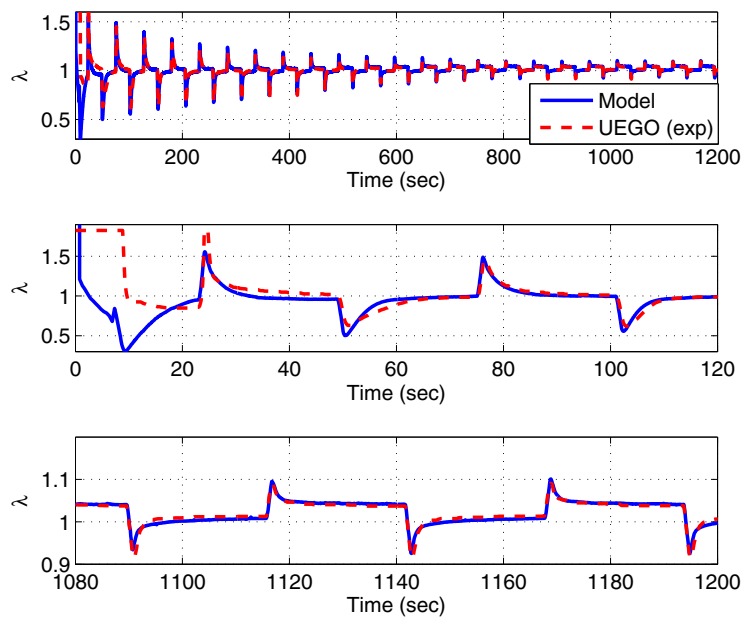

Fig. 7. Simulated and experimental $\lambda$ response for E85 $m_{i n j}(k)$, should be expressed as a function of previous states, current output and other current inputs since current states are not available. To this end, (28) can be utilized. However, the term $\sum_{i=1}^{j} m_{\text {evap }, i}(k)$ is expressed involving complicated nonlinearity in $m_{i n j}(k)$, hence causing difficulty in getting the inverted expression of $m_{i n j}(k)$ given a desired fuel in the cylinder, $m_{f, c y l}^{\text {des }}(k)$. A reasonable approximation can be utilized to remedy this problem. First, let us express $m_{\text {evap }, i}(k)$ as follows using (26) and (20):

$$
m_{\text {evap }, i}(k)=g_{i}(k) \cdot m_{p, i}^{I V O}(k),
$$

where

$$
g_{i}(k) \triangleq \min \left\{1, \quad \bar{\alpha}(k) \frac{V P_{i}(k) \times m_{p}^{I V O}(k)}{\sum_{i} V P_{i}(k) \times m_{p, i}^{I V O}(k)}\right\} .
$$

The normal vapor pressure of $i$-th component at the current step $k, V P_{i}(k)$, can be calculated using the current engine coolant temperature, $\operatorname{ECT}(k)$, and the current ethanol content, $e(k)$. Using (32) and (13), (28) is then expressed as:

$$
\begin{aligned}
m_{f, c y l}(k)= & (1-X(k)) \cdot m_{i n j}(k)+\sum_{i=1}^{j} g_{i}(k) \cdot m_{p, i}^{I V O}(k) \\
= & \left(1-X(k)+\sum_{i} g_{i}(k) \cdot X_{i}(k)\right) \cdot m_{i n j}(k) \\
& +\sum_{i} g_{i}(k) \cdot m_{p, i}(k-1)
\end{aligned}
$$

where the current total wall impacting fraction, $X(k)$, and the current wall impacting factor for each fuel component, $X_{i}(k)$, are obtained using the current ethanol content, $e(k)$, the current engine coolant temperature, $\operatorname{ECT}(k)$, and the current manifold absolute pressure, $p_{m}(k)$. To eliminate dependency of $g_{i}(k)$ on $m_{i n j}(k)$ in (34), we use a simplifying assumption that the composition of the fuel puddle is not affected significantly by the difference between the current injection, $m_{i n j}(k)$, and the previous injection, $m_{i n j}(k-1)$. This assumption is reasonable for a large puddle mass, when higher accuracy of a transient fuel compensation is required. We can then approximate $g_{i}(k)$ in (33) as:

$$
\hat{g}_{i}(k)=\min \left\{1, \hat{\bar{\alpha}}(k) \frac{V P_{i}(k) \times \hat{m}_{p}^{I V O}(k)}{\sum_{i} V P_{i}(k) \times \hat{m}_{p, i}^{I V O}(k)}\right\},
$$

where

$$
\begin{aligned}
& \hat{m}_{p, i}^{I V O}(k)=m_{p, i}(k-1)+X_{i}(k) \times m_{i n j}(k-1), \\
& \hat{m}_{p}^{I V O}(k)=\sum_{i=1}^{j} \hat{m}_{p, i}^{I V O}(k) .
\end{aligned}
$$

and

$$
\hat{\bar{\alpha}}(k)=1-\left(1+\hat{B}_{p}(k)\right)^{-\alpha(k)} .
$$

In (38), $\alpha(k)$ can be calculated using the current inputs according to (18) and the approximation of the transfer number at the current step $k, \hat{B}_{p}(k)$, can be computed as:

$$
\hat{B}_{p}(k)=\frac{\sum_{i} V P_{i}(k) \times \hat{f}_{p, i}(k)}{\widehat{P P}_{a i r}(k) \times M_{a i r} \times \sum_{i} \hat{f}_{p, i}(k) / M_{i}},
$$


where

$$
\begin{aligned}
\hat{f}_{p, i}(k) & =\frac{\hat{m}_{p, i}^{I V O}(k)}{\hat{m}_{p}^{I V O}(k)}, \\
\widehat{P P}_{a i r}(k) & =p_{m}(k)-\frac{\sum_{i} V P_{i}(k) \times \hat{f}_{p, i}(k) / M_{i}}{\sum_{i} \hat{f}_{p, i}(k) / M_{i}} .
\end{aligned}
$$

Using the approximation, (35), the transient fuel compensation is then calculated from (34) as:

$$
m_{i n j}^{T F C}(k)=\frac{m_{f, c y l}^{\text {des }}(k)-\sum_{i} \hat{g}_{i}(k) \cdot m_{p, i}(k-1)}{1-X(k)+\sum_{i} \hat{g}_{i}(k) \cdot X_{i}(k)},
$$

where

$$
m_{f, c y l}^{\text {des }}(k) \triangleq \frac{m_{\text {air }}(k)}{A F R_{s}(k)} .
$$

One easier way to approximate $g_{i}(k)$ is to use previously calculated $g_{i}$ in the puddle model so that $\hat{g}_{i}(k)=g_{i}(k-1)$ without recalculation of $\hat{g}_{i}(k)$ as above. This simpler approximation, however, results in poorer TFC performance. Fig. 8
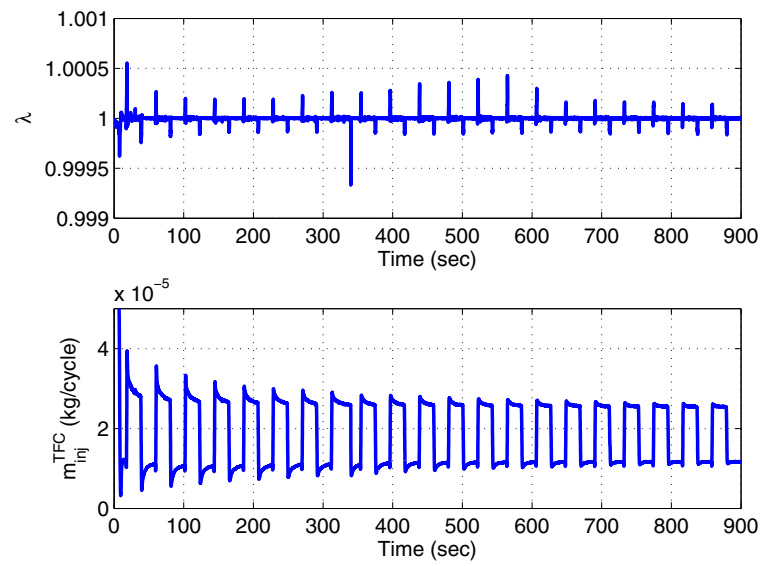

Fig. 8. Simulation of the transient fuel compensation for E22

shows the TFC simulation result to demonstrate the accuracy of our assumption and the approximation using (35)-(43). The simulation was done under the assumption that the plant follows the model perfectly and the states are known. A simulation using the model for E22 with inputs $p_{m}, m_{\text {air }}$, $E C T$ and $N$ as prescribed in the experimental data used in section $\mathrm{V}$, and $m_{i n j}$ as prescribed from the proposed TFC in (35)-(43) tuned for E22 is shown in Fig. 8. The simulated $\lambda$ trace shows several spikes due to the errors caused by the approximation but their amplitudes are negligible. This AFR accuracy is of course achieved only with very well tuned fuel puddle model parameters and known ethanol percentage.

\section{CONCLUSION}

In this paper, a physics-based fuel puddle model for any ethanol-gasoline mixture is developed. This model may be used for air-to-fuel ratio control in FFVs with engines equipped with port fuel injection. First, a multi-component fuel comprised of five chemical compounds is parameterized by ethanol content and is utilized to characterize gasoline-ethanol blends. The model consists of a droplet evaporation model and single-puddle vaporization model. The droplet evaporation model is pre-simulated off-line to generate port wall-impacting factors of the injected multi-component fuel to be used in single-puddle. A single-puddle vaporization model is then utilized as a cycle-based model that may be simulated on-line to characterize the fuel puddle dynamics in port fuel injected engines. To verify the validity of the model, simulation results are compared with a limited number of experimental data. A transient fuel compensator using the proposed model is also formulated. Since direct pole-zero cancelation is not available for the nonlinear model, an approximation is used to invert the model to design a TFC. The TFC approximation is demonstrated with a simulation.

\section{REFERENCES}

[1] C. F. Aquino, "Transient a/f control characteristics of the 5 liter central fuel injection engine," SAE paper 810494.

[2] J. J. Batteh and E. W. Curtis, "Modeling transient fuel effects with alternative fuels," SAE paper 2005-01-1127.

[3] E. W. Curtis, C. F. Aquino, D. K. Trumpy, and G. C. Davis, "A new port and cylinder wall wetting model to predict transient air/fuel excursions in a port fuel injected engines," SAE paper 961186.

[4] E. W. Curtis, S. Russ, C. Aquino, G. Lavoie, and N. Trigui, "The effects of injector targetting and fuel volatility on fuel dynamics in a PFI engine during warm-up," SAE paper 982519.

[5] R. C. Delgado, A. S. Araujo, and V. J. Fernandes Jr., "Properties of Brazilian gasoline mixed with hydrated ethanol for flex-fuel technology," Fuel Processing Technology, vol. 88, no. 4, pp. 365-368, 2007.

[6] M. L. Greenfield, G. A. Lavoie, C. S. Smith, and E. W. Curtis, "Macroscopic model of the D86 fuel volatility procedure," SAE paper 982724.

[7] L. Guzzella and C. H. Onder, Introduction to Modeling and Control of Internal Combustion Engine Systems. Springer, 2004.

[8] F. P. Incropera and D. P. DeWitt, Fundamentals of Heat and Mass Transfer, 5th ed. John Wiley \& Sons, 2002.

[9] K. Kar, T. Last, C. Haywood, and R. Raine, "Measurement of vapor pressures and enthalpies of vaporization of gasoline and ethanol blends and their effects on mixture preparation in an SI engines," SAE paper 2008-01-0317.

[10] M. Locatelli, C. H. Onder, and H. P. Geering, "An easily tunable wallwetting model for PFI engines," SAE paper 2004-01-1461.

[11] M. Locatelli, E. Alfieri, C. H. Onder, and H. P. Geering, "Identification of the relevant parameters of the wall-wetting system by extended Kalman filtering," Control Engineering Practice, vol. 14, no. 3, pp. 235-241, 2006.

[12] P. J. Maloney, "An event-based transient fuel compensator with physically based parameters," SAE paper 1999-01-0553.

[13] J. Senda, T. Higaki, Y. Sagane, H. Fujimoto, Y. Takagi, and M. Adachi, "Modeling and measurement on evaporation process of multicomponent fuels," SAE paper 2000-01-0280.

[14] W. A. Sirignano, Fluid Dynamics and Transport of Droplets and Sprays. Cambridge University Press, 1999.

[15] D. B. Spalding, Combustion and Mass Transfer. London: Pergamon Press, 1979.

[16] C. F. Taylor, Internal Combustion Engine in Theory and Practice. The MIT Press, 1985, vol. 2, revised edition.

[17] R. E. Treybal, Mass-Transfer Operations, 3rd ed. McGraw-Hill, 1980.

[18] G. F. Yao, S. I. Abdel-Khalik, and S. M. Ghiaasiaan, "An investigation of simple evaporation models used in spray simulations," Journal of Heat Transfer, vol. 125, no. 1, pp. 179-182, 2003.

[19] C. L. Yaws, Yaws' Handbook of Thermodynamic and Physical Properties of Chemical Compounds. Knover, 2003. 